\title{
Viabilité et développement durable
}

\author{
Marie-Hélène Durand ${ }^{1}$, Sophie Martin ${ }^{2}$, Patrick Saint-Pierre ${ }^{3}$ \\ ${ }^{1}$ Économie, IRD, UMR GRED (Université Montpellier 3 / IRD), 34394 Montpellier cedex 5, France \\ ${ }^{2}$ Mathématique, IRSTEA, LISC, 63172 Aubière cedex, France \\ ${ }^{3}$ Mathématique, LEDa-SDFi, Université Paris Dauphine, 75005 Paris, France
}

Au sein du dossier «Adaptation aux changements climatiques », NSS avait déjà accueilli un article de J.-P. Aubin sur une application possible de la théorie de la viabilité dans le domaine du développement et de l'environnement (NSS, 18, 3 [2010], 277-286). Dans le présent article, les auteurs reprennent cette théorie et examinent les représentations du développement durable et de leurs propriétés en matière d'équité intergénérationnelle, en refusant des approches optimales trop brutales et faiblement informatives. C'est ainsi qu'ils montrent que la théorie de la viabilité, déjà fréquemment employée dans la gestion des ressources renouvelables, permet d'introduire une flexibilité capable de prendre en compte incertitudes et contraintes, au prix d'un formalisme auquel il faut bien sûr s'accoutumer et se former. Cela devrait en faire un des outils de modélisation et d'optimisation nécessaires à l'étude des anthroposystèmes.

La Rédaction

\section{Mots-clés :}

viabilité ;

développement

durable ; équité intergénérationnelle ; optimisation

\section{Keywords:}

viability; sustainable development; intergenerational equity; optimization
Résumé - Cet article présente une utilisation possible des outils de la viabilité dans le cadre d'un modèle économique de développement durable. L'objectif de ce travail est de montrer que l'approche «viabilité » ne se réduit pas à la seule considération du respect des contraintes, mais peut servir également à traiter de problèmes d'optimisation intertemporelle. Une nouvelle façon d'évaluer l'équité intergénérationnelle est proposée dans le cadre d'un modèle théorique souvent utilisé pour l'analyse économique du développement durable.

\begin{abstract}
Viability and sustainable development. This paper presents an example of the advantages of using mathematical viability theory concepts and tools to study sustainable development issues. Instead of seeking optimal solutions according to a predefined value function, viability theory follows a inverse approach and does not postulate any a priori behaviours. Its methods and tools concern the set of all evolutions from a given situation that remain within a given set of constraints. An evolution is called viable (sustainable) if constraints are permanently satisfied. These constraints may be physical and as such imperative, or normative and consequently possibly modifiable. The aim of the paper is to show that the viability approach does not only encompass constraint satisfaction but also helps solve intertemporal optimisation problems. By applying a macroeconomic model generally used as a benchmark model to study theoretical issues of sustainable development we propose a new approach to assessing intergenerational equity. We describe two viability approaches to tackle the intergenerational equity issue: the first, proposed earlier by previous authors, concerns a minimal set of guaranteed rights for all generations; the second aims to minimize inequalities between generations while respecting resources and production constraints. Numerical computations have been performed to graphically display the solutions.
\end{abstract}

Auteur correspondant : M.-H. Durand, Marie-Helene.Durand@ird.fr 


\section{Introduction}

Les vingt dernières années témoignent d'une importante prise de conscience des problèmes environnementaux et des changements globaux induits, et non contrôlés, parl'activitéhumaine. Ces dommages environnementaux, susceptibles d'entraver la capacité des générations futures, sinon actuelles, à assurer leur bien-être sont maintenant une préoccupation première et le « développement durable », censé remédier à cette sombre perspective, est perçu comme un défi. Le point de vue de cet article concerne les modèles économiques de développement durable ; il reprend une communication faite lors d'un colloque sur les nouvelles lectures théoriques et les innovations méthodologiques de la problématique du développement durable ${ }^{1}$.

Il est fréquent de voir les mots « viable » et « durable » associés ou pris dans la même acception. La viabilité est, comme la durabilité, un concept polysémique - signifiant la capacité d'une entité à survivre -, développé en écologie (Population Viability Analysis) et en mathématiques. Nous nous référons ici à la théorie mathématique de la viabilité, consacrée à l'étude de la régulation des systèmes dynamiques sous contraintes, que nous nous proposons d'appliquer aux modèles économiques du développement durable.

La théorie mathématique de la viabilité suscite un certain engouement auprès des chercheurs en sciences de l'environnement ou en sciences sociales, mais elle s'applique aussi à bien d'autres domaines y compris la physique. Le succès de cette théorie provient de sa capacité à mobiliser des métaphores très suggestives et à représenter des concepts pertinents. À la différence de méthodes plus classiques, elle permet d'étudier les systèmes dynamiques non pas en recherchant « une solution optimale » à partir d'un critère posé a priori, mais d'abord en termes de respect des contraintes à chaque instant et de décisions prises à temps, en considérant l'adaptation des évolutions sans préjuger du futur. Les concepts de base ont été décrits dans de nombreux articles (Aubin, 1996 ; Aubin et Saint-Pierre, 2005 et 2007 ; Aubin, 2010) et l'arsenal mathématique a été développé dans plusieurs ouvrages (Aubin, 1991 et 1997 ; De Lara et Doyen, 2008 ; Aubin et $a l ., 2011$ ). Pour ce qui concerne les problèmes de développement durable, de nombreuses applications de la théorie de la viabilité ont été faites².

Dans ce travail, nous présentons quelques exemples d'utilisation des outils de la viabilité dans les modèles

\footnotetext{
1 Colloque « La problématique du développement durable vingt ans après: nouvelles lectures théoriques, innovations méthodologiques et domaines d'extension ", 20-22 novembre 2008, Clersé, Université de Lille. Ce travail a été réalisé dans le cadre du projet DEDUCTION, financé par l'ANR-ADD (20072010).
}

économiques du développement durable. Cette illustration est faite dans le cadre des modèles de croissance néoclassiques issus de celui de Ramsey (1928), destinés à étudier le mode de répartition entre les générations de l'épargne et de la consommation permettant d'atteindre un équilibre stable de croissance optimale. Le modèle de Ramsey a été repris par Hotelling (1931), Hartwick (1977), Solow (1974), Dasgupta et Heal (1974) pour explorer les conditions d'existence d'une solution en présence d'une ressource épuisable. Le développement durable est alors considéré comme une question de répartition entre générations et de possibilité, ou non, de maintenir indéfiniment un certain niveau de vie avec une quantité de ressource finie. Ce modèle canonique sert de support à de nombreuses recherches en économie sur le développement durable, conçu comme un problème de justice entre des générations qui se succèdent à l'infini et dontle « bienêtre » ne doit pas être mis en cause par les actions de générations précédentes.

La théorie de la viabilité est perçue comme une méthode prometteuse mettant l'accent d'abord sur les contraintes et leur respect, plutôt que sur la recherche d'équilibres particuliers pour des évolutions spécifiées a priori. L'objectif de cet article est de montrer que la théorie de la viabilité ne se limite pas au simple calcul d'un noyau de viabilité relatif à un système dynamique sous contraintes, mais qu'elle peut également servir à rechercher des évolutions optimales sous contraintes. Nous nous appuyons pour cette illustration sur un modèle de base qui sera progressivement complexifié. Les équations exprimant la dynamique et les contraintes de ces modèles sont décrites en annexes (Annexes A1 à A4).

Le modèle économique standard de développement durable est brièvement exposé dans la première section. Nous présentons, en deuxième section, le travail de Martinet et Doyen (2007), qui ont été les premiers à utiliser la théorie de la viabilité dans le cadre du modèle de Dasgupta-Heal et Solow pour définir le développement durable comme le respect en tout temps d'un ensemble de contraintes, en introduisant une extension à ce travail où la population peut fluctuer sous l'effet d'un contrôle endogène. Nous abordons ensuite le problème de l'équité entre générations avec deux approches: garantir un ensemble de « droits minimum » à toutes les

\footnotetext{
2 Parmi une abondante littérature dans le domaine de la gestion des ressources renouvelables, voir par exemple : Aubin et Saint-Pierre, 2007 ; Baumgärtner et Quaas, 2009 ; Béné et al., 2001 ; Béné et Doyen, 2008 ; Bonneuil, 2003 ; Doyen et Béné, 2003 ; Doyen et al., 2007 ; De Lara et al., 2007 ; Eisenack et al., 2006 ; Martinet et al., 2007 ; Rapaport et al., 2006 ; Tichit et al., 2007. Dans le domaine du changement climatique, voir : Aubin et al., 2004 ; Tichit et al., 2004 ; Bernardo, 2008 ; Doyen et al., 2008. Pour ce qui concerne la résilience écologique, voir : Martin, 2004 et 2005. Pour ce qui concerne la démographie, voir : Bonneuil, 2000 ; Bonneuil et Saint-Pierre, 2008.
} 
générations en agissant sur les contraintes et minimiser les inégalités entre générations, tout en respectant l'ensemble des contraintes en agissant sur la dynamique du système. Enfin, nous présentons la manière dont les outils de la théorie de la viabilité permettent de sélectionner une évolution optimale.

\section{Le modèle économique standard de développement durable}

Dans le modèle d'accumulation de capital et d'épuisement de ressource, utilisé par Dasgupta-Heal et Solow, le processus de production nécessite à la fois du capital reproductible $(K)$ et du capital non reproductible $(S)$, dont on extrait une quantité $R$ à chaque période (Annexe A1). La production est soit consommée $(C)$, soit réinvestie $(d K / d t)$. C'est un modèle de croissance à deux biens où il s'agit d'arbitrer entre investissement et consommation sous contraintes de ressources. Le problème consiste à trouver la solution qui maximise le bien-être global de l'ensemble des générations d'ici à l'infini, selon un principe d'équité. Il est nécessaire pour cela d'avoir un critère d'optimisation intertemporel donnant une mesure de l'équité entre générations. Le plus courant de ces critères d'équité intergénérationnelle est la somme escomptée des utilités d'une infinité de générations représentant la fonction intertemporelle de bien-être social. Les fondements éthiques de ce critère d'équité intergénérationnelle ont été justifiés par Koopmans (1960), mais celui-ci reste controversé en raison des problèmes posés par le choix du taux d'actualisation et le traitement inéquitable des générations que cela implique. Il est pourtant utilisé dans la plupart des situations où l'on cherche à évaluer les impacts économiques de long terme ; c'est, par exemple, le cas dans le rapport Stern sur l'impact économique du changement climatique, où cet auteur a choisi délibérément et a priori un taux d'actualisation proche de zéro, suscitant nombre de critiques. De nombreuses extensions du modèle de base ont été proposées. Quelles que soient les variantes de ce modèle, aussi compliquées soient-elles, elles servent à explorer les propriétés d'une trajectoire particulière sélectionnée a priori selon un critère d'équité, et les résultats obtenus dépendent du critère d'optimisation choisi. En utilisant le critère de Koopmans (maximisation de la somme infinie des utilités escomptées), il a été montré qu'il n'est pas possible de maintenir indéfiniment le niveau de consommation et que celui-ci tend vers zéro à moins que l'accumulation de capital ou le changement technique ne compensent l'extraction des ressources. Le développement durable n'est possible que dans le cas particulier où le capital reproductible se substitue au capital naturel non reproductible (par exemple en utilisant une fonction de production de type CobbDouglas : voir Annexe A1). En se référant à la notion de justice établie par Rawls (1987) dans un cadre intratemporel, Solow (1974) a montré que l'utilisation du critère du «maximin" (sélectionnant l'évolution qui maximise le bien-être de la génération ayant le plus faible niveau de bien-être) impliquait un niveau de consommation constant à travers les générations.

Cette approche économique qui définit le développement durable comme un problème d'équité entre générations est en accord avec la définition du rapport Brundtland et n'a pas l'ambition de représenter les nombreuses autres questions de développement durable. Dans cet article, nous ne discuterons pas le bien-fondé de ce modèle, qui a été conçu seulement pour étudier le problème de la répartition en horizon infini avec une ressource finie. C'est à ce titre que, à l'instar de nombreux auteurs, nous le reprenons pour illustrer, dans un cadre connu, l'utilisation des outils de la viabilité et la façon de trouver une solution équitable optimale, alternative à des critères d'équité contestés.

\section{Durabilité (viabilité) et respect des contraintes}

La théorie de la viabilité est une théorie mathématique basée sur l'analyse multivoque, elle réalise des calculs d'ensembles et manipule des fonctions d'ensembles. Un des ensembles fondamentaux de cette théorie est le noyau de viabilité : ensemble des états initiaux à partir desquels part au moins une évolution qui respecte toujours un ensemble de contraintes. Ces contraintes, dites de viabilité, reflètent le problème étudié et sont définies dans le cadre d'un modèle censé représenter formellement ce problème. Les résultats mathématiques apportés par la théorie de la viabilité autorisent ces contraintes à dépendre du temps et des variables d'état. Au lieu de rechercher directement les solutions d'une fonction objectif définie préalablement, se limitant ainsi à une évolution particulière dont on ne peut garantir la réalisation soumise à un avenir inconnu, la théorie de la viabilité adopte une démarche inverse et ne postule pas d'objectif a priori. Elle s'intéresse à l'ensemble de toutes les évolutions envisageables à partir d'un état présent. Il s'agit en premier lieu de distinguer, parmi cet ensemble des possibles offert à un moment donné, les évolutions viables de celles qui ne le sont pas pour des contraintes et une dynamique données, puis ensuite de rechercher, à l'aide de règles de rétroaction, la manière de «piloter » le système pour sélectionner les évolutions dotées de propriétés souhaitées. En calculant le noyau de viabilité relatif à un ensemble de contraintes et à une dynamique donnés, on peut savoir s'il existe, ou non, des possibilités d'évolutions viables à partir d'une situation initiale particulière et déterminer les régulations nécessaires pour qu'une évolution viable puisse le rester en fonction de l'évolution 
des variables d'état du système. Tout le problème consiste à définir convenablement ces contraintes et cette dynamique d'évolution, c'est-à-dire à concevoir un modèle aussi pertinent que possible pour répondre à une question donnée. Dans les modèles économiques de développement durable, ces contraintes disent, par exemple, qu'on ne peut consommer durablement plus que ce qui est produit, ou que les possibilités d'exploitation d'une ressource épuisable sont limitées par le stock des réserves existantes, ou que l'exploitation d'une ressource naturelle renouvelable ne doit pas dépasser sa capacité de renouvellement, ou encore qu'il est nécessaire de garantir à toutes les générations un certain niveau de vie minimum par habitant.

Une évolution est dite viable (durable) si elle respecte en permanence ces contraintes de viabilité (durabilité), qui peuvent être physiques - et elles sont alors impératives - ou normatives - donc éventuellement modifiables. Ces contraintes, qui déterminent les conditions de viabilité du système, peuvent être renforcées si l'on souhaite examiner la viabilité d'une " politique plus restrictive », ou relâchées si le noyau de viabilité est vide et qu'il n'existe aucune possibilité d'évolution viable dans ces conditions. Les évolutions ont une dynamique régie par un certain nombre de paramètres. Certains peuvent être constants, et ce sont alors des coefficients. D'autres peuvent être amenés à évoluer pour maintenir la viabilité du système, lorsqu'ils dépendent du temps ou des variables d'état, et ce sont alors des contrôles ou des régulons. Quand on se trouve au départ dans le noyau de viabilité, évoluer dans une mauvaise direction et ne plus être en mesure de toujours respecter les contraintes de viabilité est une situation qui peut éventuellement se produire. Dans une telle situation, il est nécessaire de modifier son comportement en temps opportun afin de rester dans le noyau de viabilité ou sur sa frontière et d'éviter une crise à venir. Savoir quand et comment les contrôles doivent être modifiés estl'objet de la recherche en théorie de la viabilité, et des techniques ont été mises au point pour répondre à ces questions.

Martinet et Doyen (2007) ont appliqué les outils de la théorie de la viabilité au modèle de Dasguspta-Heal et Solow et ont défini la durabilité par le fait de pouvoir respecter des contraintes en permanence, ce qui consiste à rechercher les conditions dans lesquelles le noyau de viabilité n'est pas vide. Ces auteurs retrouvent les résultats précédemment acquis, qu'ils généralisent ; mais surtout, ils montrent que, lorsque le noyau de viabilité n'est pas vide, les évolutions durables ne sont pas uniques et ne se réduisent pas à une évolution optimale, alimentant le débat sur les relations entre «optimalité » et «durabilité ». Ils imposent des contraintes plus restrictives au noyau de viabilité en stipulant que les niveaux de consommation et le stock de ressources épuisables ne devront jamais être inférieurs à certains seuils admissibles.

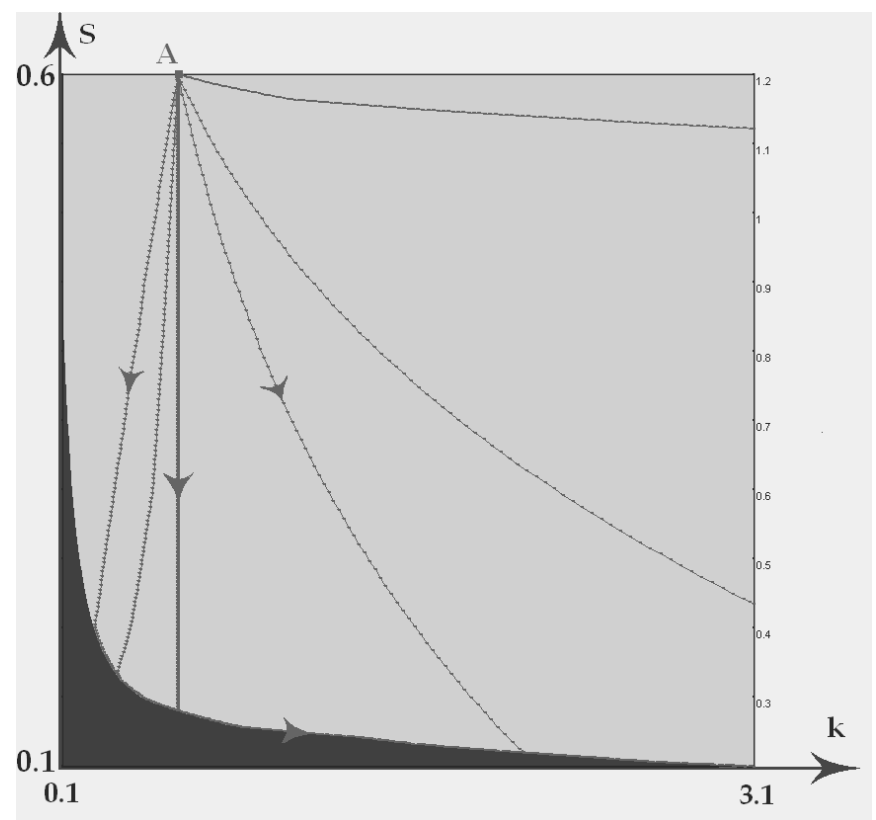

Fig 1. Noyau de viabilité du modèle A2.3.

Cela signifie, pour une société dont les conditions initiales se situent dans le noyau de viabilité, que celle-ci peut garantir l'existence d'une quantité de ressource minimum et un niveau minimum de consommation à toutes les générations futures. Les auteurs définissent alors la durabilité comme la possibilité de transmettre un ensemble de " droits minimaux » à toutes les générations. Nous avons également repris le modèle de Dasgupta-Heal et Solow (Annexe A1) comme cas d'étude et étendu l'approche « viabilité » de Martinet et Doyen (Annexe A2.1) en prenant en compte les variations de population, l'explosion démographique du siècle passé étant un élément central des préoccupations actuelles sur le développement durable (Annexes A2.2 et A2.3).

La figure 1 présente un noyau de viabilité calculé de façon numérique (Saint-Pierre, 1994 et 1997), où, comme dans la proposition de Martinet et Doyen (2007), la consommation par habitant (variable de contrôle) et le stock de la ressource épuisable par habitant (variable d'état) ne peuvent descendre en dessous d'un certain seuil. Alors que, dans ce type de modèle, la population est généralement considérée comme constante (Annexe A2.1), ou croissante mais de façon exogène (Annexe A2.2), ce noyau de viabilité a été calculé pour des variables par habitant avec un système dynamique associé permettant de contrôler les fluctuations de population (Annexe A2.3). Dans ce modèle, la contrainte « comptable " porte sur la consommation totale, qui ne peut être supérieure à ce qui est produit globalement ; le montant de capital par tête peut donc être amené à diminuer si la croissance démographique ou/et la consommation par habitant sont trop élevées. La possibilité 

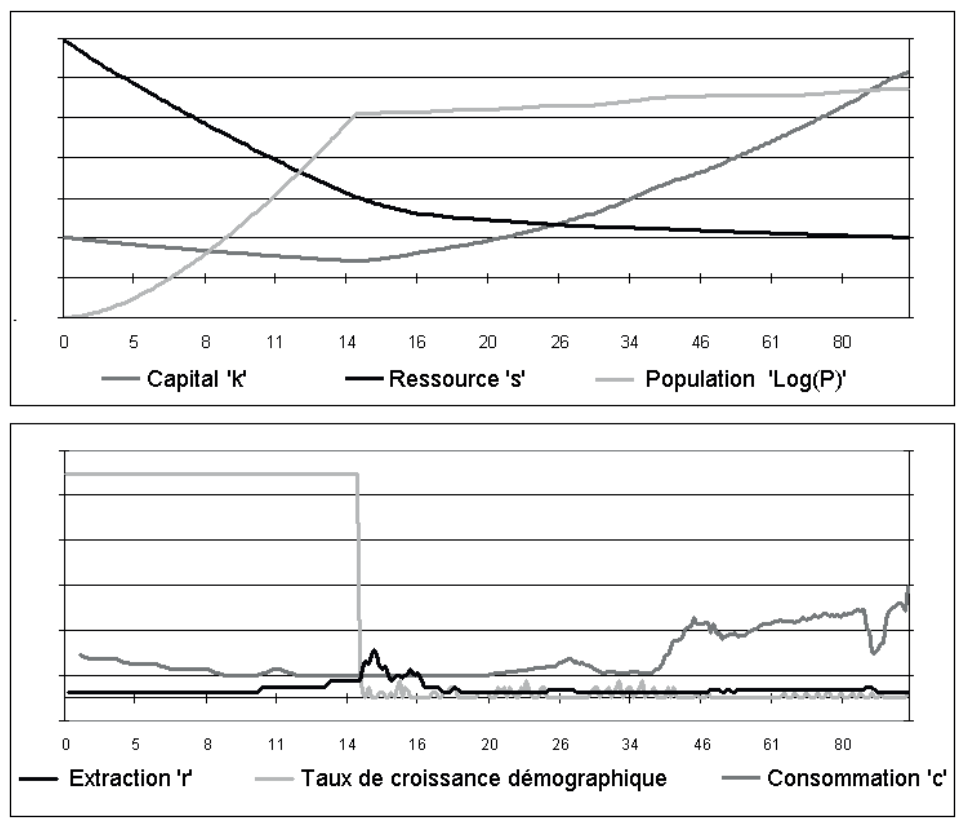

Fig 2. Évolution des variables d'état (graphe du haut) et de contrôle (graphe du bas) d'une trajectoire viable (modèle A2.3).

d'endogénéiser les mouvements de population est un nouvel exemple de l'apport des outils de la viabilité à ce modèle. Le taux de croissance de la population est un paramètre de régulation (ou contrôle) contraint d'évoluer entre deux bornes, minimale et maximale, dont l'écart a été choisi a priori mais établi de façon relativement large. La borne inférieure a été fixée à $n_{\min }=0$ (excluant une dépopulation) et la borne supérieure à $n_{\max }=0,1$ (autorisant une croissance de population de $10 \%$ au maximum à chaque génération). La fonction de production est fixe de type Cobb-Douglas $\left(K^{\alpha} R^{\beta}\right.$ avec $\alpha=3 / 4$ et $\left.\beta=1 / 4\right)$ et il n'y a pas de progrès technique dans cette illustration.

La forme du noyau de viabilité dépend de la dynamique du système. Dans ce modèle particulier où la fonction de production permet la substitution entre ressource épuisable et capital manufacturé, il est possible d'être viable en disposant au départ uniquement de ressource ou uniquement de capital, à condition que ces quantités soient infiniment grandes. Le point A (Fig. 1) représente une situation de départ, en ressource et capital, à partir de laquelle la ressource ne peut que diminuer. À partir de cette situation initiale viable, un grand nombre d'évolutions sont possibles selon les différentes combinaisons de contrôles (extraction, consommation et croissance de la population), qui sont choisis à chaque moment par les différentes générations.

La figure 2 montre un exemple des trajectoires suivies par les variables d'état - le capital manufacturé par personne $(k)$, la quantité de ressource disponible par personne $(s)$ et le niveau de population $(P)$ - en fonction des décisions de chaque génération (consommation et quantité de ressource extraite par habitant, taux de croissance démographique) pour que l'évolution particulière issue $\mathrm{du}$ point $\mathrm{A}\left(k_{0}, s_{0}, P_{0}\right)$ de la figure 1 reste contenue dans le noyau de viabilité. Dans cet exemple, la situation initiale est celle d'une économie sousdéveloppée, faiblement capitalistique, mais avec une importante dotation en ressource naturelle et un taux de croissance démographique élevé.

Durant les premières générations, la population augmente très fortement et la production, qui dépend essentiellement de l'exploitation de la ressource naturelle, n'est pas suffisante pour maintenir le niveau de consommation par tête. Ce mode de développement, qui entraîne une diminution rapide de la ressource sans que cette extraction soit réinvestie pour permettre l'augmentation de capital, n'est pas durable. Le changement se produit à la quatorzième génération, qui réduit drastiquement son taux de croissance démographique (ou qui émigre) tout en augmentant ponctuellement la quantité de ressource extraite par habitant, permettant ainsi d'augmenter le niveau de capital par tête. Cela permet d'amorcer un changement de mode de production où le capital reproductible se substitue peu à peu au capital non reproductible et d'initier une période de croissance continue, bien que la ressource ait atteint son niveau de gel. Il devient alors possible d'augmenter « durablement » le niveau de consommation par habitant.

\section{Durabilité (viabilité) et équité}

Considérer le développement durable comme l'obligation de respecter des contraintes de ressources, et d'en 
épargner une partie pour assurer la transmission d'une quantité minimale de biens aux générations futures, n'est pas suffisant. Le noyau de viabilité calculé précédemment contient toutes les situations initiales à partir desquelles il existe au moins une évolution viable et donc des possibilités de contrôles tels que les contraintes pourront toujours être respectées. Il peut exister un grand nombre d'évolutions viables et, parmi celles-ci, beaucoup ne sont pas des évolutions « équitables ». Si certaines générations se trouvent, par exemple, dans une situation où le niveau de la ressource est infiniment grand par rapport à leurs besoins, rien a priori ne peut leur indiquer la nécessité de ne pas la gaspiller, encore moins de l'épargner ou d'investir pour les générations futures. Ce n'est que lorsque le caractère épuisable des ressources est perçu que s'opère cette prise de conscience. Décidés trop tard, les nécessaires changements de comportement imposeront aux générations suivantes des sacrifices qui, s'ils avaient été mieux répartis sur l'ensemble des générations, auraient pu être moindres. Cette situation sera cependant viable si les générations sacrifiées réalisent, au détriment de leur propre bien-être, les efforts nécessaires pour respecter les contraintes de viabilité. Il importe donc de rechercher, parmi les évolutions viables, celles qui offriront une plus juste répartition des choix de consommation et des efforts d'investissement, en fonction des dotations et des capacités de chaque génération, de façon à ce qu'aucune d'entre elles ne soit injustement sacrifiée.

Il y a différentes manières d'aborder ce problème avec les outils de la viabilité, et nous en présenterons deux : la garantie des droits et la réduction des inégalités. La première, envisagée par Martinet et Doyen (2007), consiste à agir sur les contraintes et à rechercher le niveau maximum de «droits minimum », en termes de consommation garantie par habitant et de ressource préservée, qui pourront être transmis à l'ensemble des générations : contraindre, à toutes les périodes, les générations présentes à respecter les «droits » des générations futures (Annexe A2.3). Nous nous concentrerons sur la seconde, qui consiste à utiliser une fonction qui permettra de sélectionner, parmi toutes les évolutions viables, celle pour laquelle l'inégalité entre générations consécutives sera la plus faible. Pour cet exercice, nous nous intéressons aux différences de consommation par habitant entre générations (Annexe A3).

Aborder le problème de l'équité entre générations consiste à disposer d'une mesure d'équité permettant de comparer et classer des suites infinies de consommation en tenant compte de l'intérêt de chaque génération. Ce problème est plus difficile que celui de l'équité intragénérationnelle, car les transferts entre générations sont univoques et l'horizon infini. Une approche normative du développement durable a été développée qui caractérise l'équité intergénérationnelle par le respect de deux axiomes : le principe de Pareto, ou d'efficience (toute suite infinie dont les éléments sont supérieurs à ceux d'une autre doit lui être préférée) ; et le principe d'anonymat faible, ou de neutralité (une permutation de n'importe quelle paire d'éléments d'une suite ne doit pas changer son évaluation). Le respect de ces deux axiomes, qui impliquent absence de gaspillage et de discrimination d'une génération quelconque, est qualifié de principe de Suppes-Sen (Asheim, 2010). En horizon infini, il n'existe pas de critère numériquement représentable satisfaisant conjointement les conditions de ces deux axiomes. La somme infinie des utilités escomptées, critère d'évaluation intertemporel le plus utilisé, respecte le principe de Pareto mais pas celui d'anonymat et ne traite pas les différentes générations de manière égalitaire (Heal, 2005 ; Asheim, 2005 et 2010).

Avoir un objectif d'équité intergénérationnelle dans un modèle de viabilité consiste à introduire un "contrôle » permettant de réguler le comportement de chaque génération (et non plus à comparer un score d'équité, issu d'un critère construit a priori, réalisé le long de différents chemins de développement par une succession infinie de générations), de façon à ce qu'aucune d'entre elles, le long du développement, ne soit une génération «injustement » sacrifiée en tant que telle par des choix présents ou précédents. Dans le modèle simple pris comme support de cet exercice, chaque génération fait des choix entre consommer, produire et se reproduire selon ses dotations en capital naturel et manufacturé et ses besoins liés au niveau de sa population. Le résultat de ces choix, en termes d'accumulation de capital, détermine l'éventail des possibilités offertes aux générations suivantes. Sans progrès technique et avec une fonction de production autorisant la substitution entre les facteurs de production (la ressource naturelle et le capital manufacturé), le niveau de consommation et la croissance démographique sont les deux éléments qui décident de l'héritage laissé aux générations suivantes. Nous avons donc retenu le niveau de consommation par habitant et considéré queles variations de consommation par habitant entre générations successives constituaient une mesure de l' « inégalité entre les générations » qu'il faut maintenant chercher à réduire (Annexe $\mathrm{A} 3$ ). Nous avons utilisé le pourcentage de variation $\frac{d c(t) / d t}{c(t)}$, une mesure relative, plutôt que les écarts de consommation par habitant entre générations successives $d c(t) / d t$, une mesure absolue, pour tenir compte de l'effet d'accumulation de richesses le long des générations et ainsi mieux appréhender la notion de « bien-être ". C'est en effet la position des utilitaristes, qui, pour évaluer de façon équivalente le niveau de bien-être de différentes générations, emploient la fonction d'utilité, une fonction de la consommation concave et croissante permettant de donner moins de poids à l'augmentation marginale de consommation des générations les plus riches par rapport à celle des générations les plus pauvres. 


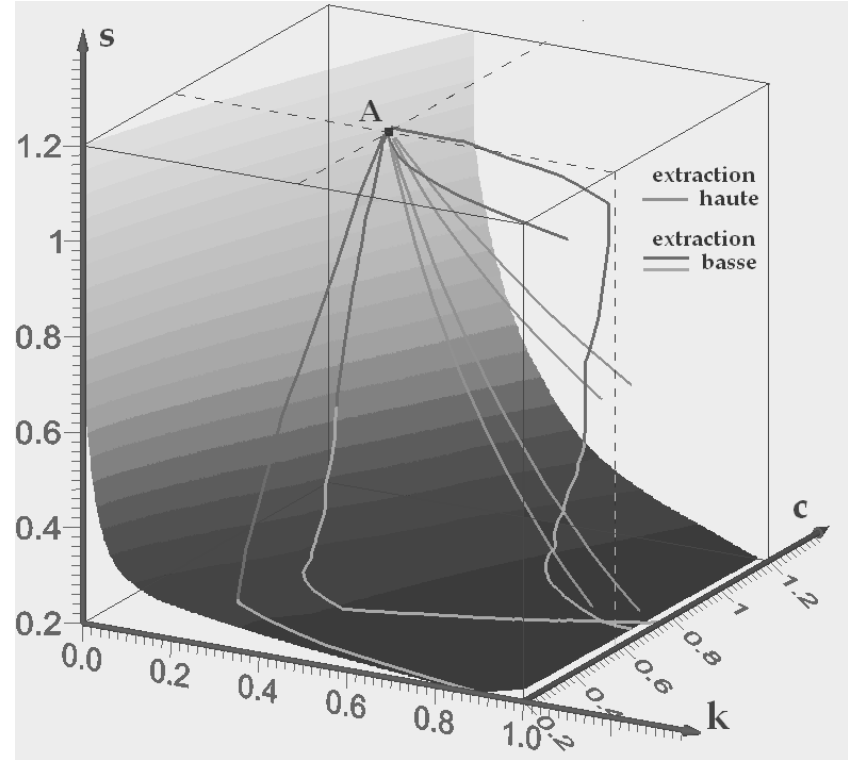

Fig 3. Noyau de viabilité du modèle A3.

Contrôler le taux de croissance de la consommation par habitant de chaque génération, tout en respectant l'ensemble des autres contraintes de viabilité, consiste à ajouter au problème précédent une dynamique auxiliaire portant sur la dérivée dans le temps de la consommation par habitant. Le fait de soumettre la consommation, qui dans l'exercice précédent était un contrôle, à l'exercice d'un nouveau contrôle, qualifié de métacontrôle, change le statut de cette variable. Elle passe formellement du statut de variable de contrôle à celui de variable d'état, ce qui augmente la dimension du système. La description de ce modèle est présentée dans l'annexe A3. Comme dans le cas précédent, le taux de croissance de la population est endogène ; c'est un paramètre de régulation qui régit le niveau de la population de façon à ce que, conjointement à l'action des autres paramètres de régulation (niveau d'extraction de la ressource naturelle et contrôle de la consommation par tête), l'évolution respecte l'ensemble des contraintes de viabilité en restant à l'intérieur ou sur la frontière du noyau de viabilité.

L'évolution du niveau de consommation par habitant est maintenant déterminée par la dynamique $d c(t) / d t=$ $v(t) c(t)$. Établir une forme d'équité intergénérationnelle en limitant les inégalités entre générations tout en respectant les contraintes écologiques et matérielles de production consiste, dans cet exemple, à contraindre $v(t)$, le taux de croissance de la consommation par habitant, à être toujours supérieur ou égal à un certain seuil minimum $v_{\text {min }}$. Si le noyau de viabilité de ce métasystème (Annexe A3) n'est pas vide, il sera possible de trouver un chemin de développement viable et équitable à partir de toute situation initiale appartenant à ce noyau. Le taux de croissance de la consommation par habitant d'une génération quelconque $v(t)$ peut être amené à diminuer ; mais, quelles que soient les générations concernées, cette diminution sera toujours limitée par la contrainte inférieure $v(t) \geq v_{\text {min }}$. Cette contrainte s'applique à toutes les générations et, couplée aux autres contraintes, elle interdit les modes de développement où le comportement de certaines générations auraient un impact trop négatif sur la consommation des générations futures. Si $v_{\min }$ est nul, la consommation par habitant des générations futures ne sera jamais inférieure à celle des générations précédentes. Si $v_{\text {min }}$ est négatif, cela signifie que la consommation par habitant pourra diminuer pour certaines générations, mais jamais en dessous d'un certain pourcentage admissible. Si $v_{\text {min }}$ est positif, cela signifie que la consommation par habitant augmentera de façon exponentielle avec les générations (le noyau de viabilité sera vide s'il n'est pas possible de respecter l'ensemble des contraintes dans ce cas). Nous n'avons pas introduit de borne supérieure $v_{\max }$, considérant qu'il n'était pas justifié de limiter la croissance des consommations futures par une contrainte a priori autre que celles portant sur la capacité de production. L'évolution du niveau de consommation par habitant est seulement contrainte par la règle $v(t) \geq v_{\text {min }}$ - un devoir moral envers les générations futures qui s'applique à toutes les générations.

La figure 3 présente la frontière inférieure de ce nouveau noyau de viabilité. Il contient l'ensemble des situations initiales à partir desquelles il existe des évolutions qui respecteront à la fois les contraintes du précédent modèle et la forme d'équité entre les générations définie plus haut. Il a été calculé en imposant un frein de $5 \%\left(v_{\min }=-0,05\right)$ au pourcentage de baisse éventuelle du niveau de consommation par tête. Quelques évolutions viables susceptibles de partir du point de dotation initiale $\mathrm{A}:\left(k_{0}, s_{0}, c_{0}, P_{0}\right)$ sont représentées. Elles diffèrent selon les diverses combinaisons de décisions sous contraintes qui peuvent être prises (consommation, extraction, croissance démographique). De nombreux choix sont admissibles; ils peuvent rester inchangés ou être modifiés à tout moment, mais ce n'est que lorsqu'une évolution s'apprête à quitter le noyau de viabilité que la modification des contrôles qui la régissent devient impérative pour rester dans le noyau de viabilité ou sur sa frontière. La valeur des contrôles est sélectionnée en fonction de celles prises par les variables d'état selon une règle en feedback calculée numériquement de façon à ce que l'évolution reste viable.

La figure 4 reprend une de ces évolutions, la deuxième à partir de la gauche figurée sur le noyau, montrant comment les contrôles ont été modifiés en fonction des valeurs prises par les variables d'état. Cette figure présente, dans la partie supérieure, le décours temporel des variables d'état (consommation par habitant : $c(t)$, capital manufacturé par habitant : $k(t)$, quantité de ressource disponible par habitant : $s(t)$, niveau de population : $P(t))$ et, dans la 

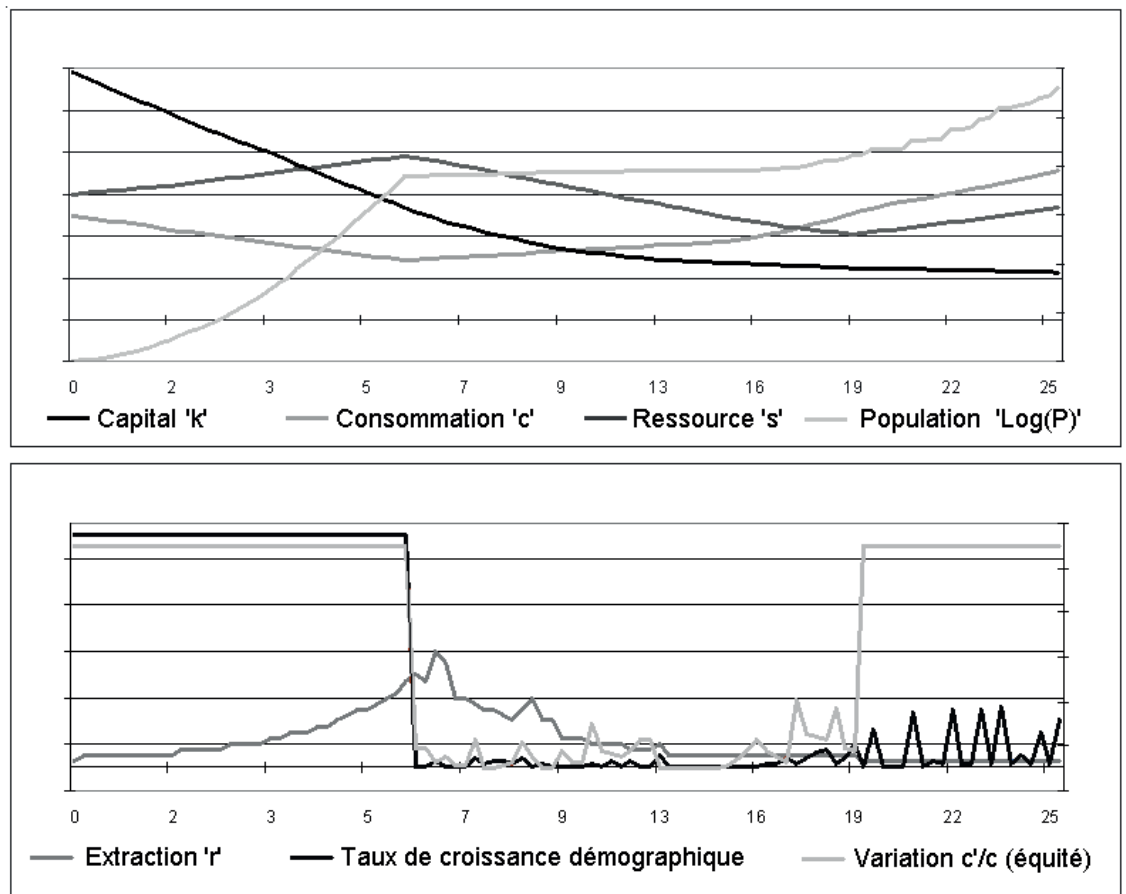

Fig 4. Évolution des variables d'état et de contrôle d'une trajectoire viable (modèle A3).

partie inférieure, le décours temporel des contrôles exercés (« équité»: $v(t)$, croissance démographique : $n(t)$, quantité de ressource extraite $r(t))$. Ce mode de développement est caractérisé à ses débuts par une forte croissance de la population, couplée à une augmentation de la consommation par habitant. Ce comportement « égoïste » des premières générations entraîne une décapitalisation, une diminution rapide de la ressource naturelle disponible, et la frontière du noyau de viabilité est rapidement atteinte. Pour pouvoir rester viable, la population de cette société doit se stabiliser et le niveau de consommation par tête est obligé de décroître. Les efforts que les générations de cette époque d'austérité sont amenées à faire sont cependant adoucis, car toutes les générations sont également obligées de respecter une limite aux baisses éventuelles de consommation par habitant (dans cet exemple, une contrainte de viabilité impose que la consommation par tête ne pourra jamais diminuer de plus de $5 \%$ ). En fin d'ajustement, le capital accumulé est suffisamment important pour que la consommation par habitant soit à nouveau en mesure de croître pour les générations suivantes, autorisant même une augmentation de population.

L'exemple de la figure 4 est issu du même point initial que celui présenté dans la figure 2 . Il possède les mêmes configurations en termes de dotations initiales et de contrôles exercés au départ. La seule différence entre ces deux graphiques consiste à avoir imposéà toutes les générations une contrainte supplémentaire réduisant les inégalités de consommation entre générations. L'introduction de cette contrainte a eu pour effet de raccourcir la période initiale de forte croissance démographique et de décapitalisation. La croissance démographique diminue ici à partir de la sixième génération, alors que, sans contrainte d'équité(Fig. 2), l'augmentation de population se poursuit pendant quatorze générations, aggravant la situation des générations suivantes. L'époque où le niveau de consommation par habitant peut augmenter est également plus précoce et, s'il existe, dans les deux cas, une succession de générations sacrifiées, en charge du réajustement, celles-ci sont moins nombreuses lorsqu' on impose une condition d'équité intergénérationnelle.

Les exemples d'évolution viable présentés sur les figures 2 et 4 , issus d'un calcul numérique, sont donnés à titre d'illustration pour montrer visuellement les fluctuations des variables d'état et de contrôle dans le cadre d'un modèle simple. Ils résultent $d^{\prime}$ un tirage au hasard à partir d'évolutions viables issues d'un point du noyau de viabilité du modèle décrit dans l'annexe A3. Le noyau de viabilité et les évolutions viables qui partent d'une situation initiale à l'intérieur du noyau dépendent du modèle choisi, de sa dynamique et des contraintes. Ils dépendent aussi de la valeur des paramètres qui ont été fixés ici de façon cohérente mais arbitraire, en particulier le niveau des bornes minimales et maximales des variables de contrôle (la consommation ou sa variation, l'extraction de ressource et le taux de croissance démographique). D'autres valeurs de ces paramètres, mais aussi d'autres 
fonctions de production, peuvent être explorées. On peut également envisager la construction d'autres « critères " agissant sur les variables d'état concernant, par exemple, la gestion et la préservation du capital naturel, ou agissant sur la dynamique de production en mettant en œuvre le progrès technique, dont on peut concevoir que l'apparition se fasse par saut en cas de crise. Dans le modèle de base que nous avons retenu, la fonction de production est à technologie constante, une hypothèse qui en limite l'intérêt. Introduire une évolution endogène du progrès technique est possible et ne modifie pas la façon de prendre en compte l'équité intergénérationnelle. Par contre, cela ajoute une dimension supplémentaire au problème de viabilité que les logiciels de calcul actuels ne permettent pas de traiter. Nous avons choisi ici de présenter cette notion d'équité en l'accompagnant d'une illustration graphique et donc privilégié la possibilité de calcul des noyaux de viabilité des modèles plus simples décrits en annexe.

\section{Durabilité (viabilité) et « optimalité 》}

Un des attraits de la théorie de la viabilité est la perspective qu'elle offre de pouvoir analyser les systèmes dynamiques sans nécessairement mettre en avant la recherche de solutions optimales selon un critère particulier, comme c'est le cas avec la théorie du contrôle optimal. En ce sens, l'approche "viabilité » et l'approche «optimalité » sont différentes, mais elles ne sont pas non plus en opposition, comme cela est dit parfois, car les outils de la viabilité permettent également de résoudre des problèmes d'optimisation, intertemporelle en particulier. Nous en donnons un exemple en poursuivant l'analyse qui a été faite de l'équité intergénérationnelle.

Pour cela, nous introduisons la fonction $\mathrm{d}^{\prime}$ inertie $^{3}$, qui permet de sélectionner la plus « équitable » possible de toutes les évolutions viables : celle où le pourcentage de variation relative du niveau de consommation par habitant entre deux générations quelconques sera le plus faible en valeur absolue. Si $k_{0}$, $s_{0}$ et $c_{0}$ sont les niveaux initiaux de capital, de ressource épuisable et de consommation par tête, $P_{0}$ étant la taille de la population, cette valeur s'écrit :

$$
\beta\left(k_{0}, s_{0}, c_{0}, P_{0}\right):=\operatorname{Inf}_{k(.), s(.), c(.), P(.))} \operatorname{Sup}_{t \geq 0}\left\|\frac{d c(t) / d t}{c(t)}\right\|
$$

La fonction $\beta($.) détermine le plus petit taux de croissance de la consommation par habitant qu'il est possible

\footnotetext{
3 Une présentation complète des propriétés mathématiques de la fonction d'inertie et du lien avec les problèmes d'optimisation est faite dans Aubin et al. (2011). Son utilisation dans des problèmes d'environnement est développée dans Aubin et al. (2004) et Aubin et Saint-Pierre (2007).
}

de ne jamais dépasser en valeur absolue pour au moins une évolution viable. Tout le long de cette évolution «la plus équitable», les variations de consommation par habitant vérifieront toujours la relation $\left\|\frac{d c(t) / d t}{c(t)}\right\| \leq \beta\left(k_{0}, s_{0}, c_{0}, P_{0}\right)$ pour toutes les générations (indexées ici par $t$ ). Théoriquement, les valeurs prises par cette fonction ne peuvent être que positives ou nulles. $\mathrm{Si}$, à partir d'un état initial donné, il s'avère que ce plus petit écart - la valeur de la fonction $\beta($.) - est nul, cela signifie que l'évolution viable la plus équitable sera celle où la consommation par tête restera constante pour toutes les générations. Dans ce modèle avec fluctuation de population, cela peut correspondre à une situation malthusienne où la croissance de la population absorbe l'augmentation de capital, ne permettant pas d'augmenter le niveau de consommation par tête, et où une baisse éventuelle de capital (en raison, par exemple, de la raréfaction des ressources) entraînera une diminution de la population et non de la consommation par tête (bloquée à son niveau initial). Cette évolution à consommation constante instaure certes une égalité entre les générations (en termes de niveau de consommation par habitant), mais exclure les possibilités d'une amélioration du niveau de vie est difficilement acceptable comme conception du développement durable et de l'équité intergénérationnelle. Ce cas particulier, où il serait possible de trouver $\beta\left(k_{0}, s_{0}, c_{0}, P_{0}\right)=0$, correspond à la solution que l'on obtient en utilisant le critère du maximin dans un cadre intergénérationnel, solution étudiée et contestée par Solow (1974) au motif qu'une société pauvre au départ serait condamnée à le rester éternellement. L'objectif de cet auteur, qui dans ce travail s'est voulu " plus rawlsien que Rawls ", était de plaider pour un rejet de ce critère rawlsien dans un cadre intergénérationnel. C'est pourquoi, comme cela a déjà été fait dans la section précédente, nous proposons de n'imposer une limite qu'aux variations à la baisse de la consommation par habitant, considérant que la notion d'équité intergénérationnelle ne doit pas empêcher l'augmentation de bien-être des générations futures. Nous suggérons pour cela d'utiliser une nouvelle fonction : la fonction d'équité. Au lieu de rechercher la valeur $\beta\left(k_{0}, s_{0}, c_{0}, P_{0}\right)$ encadrant les fluctuations de consommation par habitant entre les générations de façon telle que $\frac{d c(t) / d t}{c(t)} \leq \beta\left(k_{0}, s_{0}, c_{0}, P_{0}\right)$ en cas de hausse et $\frac{d c(t) / d t}{c(t)} \geq \beta\left(k_{0}, s_{0}, c_{0}, P_{0}\right)$ en cas de baisse, il s'agit de ne prendre en compte que la borne inférieure $v_{\min }$ pour que le taux de croissance de la consommation par habitant de chaque génération $v(t)=\frac{d c(t) / d t}{c(t)}$ soit toujours supérieur à $v_{\min }$ pour toutes 
les générations. Cette obligation envers les générations futures s'applique à toutes les générations, la génération présente et toutes celles à venir.

La fonction d'équité permet de calculer la plus grande valeur de cette borne inférieure qui peut être obtenue à partir de chaque situation initiale. Le métasystème permettant de calculer la fonction d'équité est présenté dans l'annexe A4. La valeur de la fonction d'équité au point $\left(k_{0}, s_{0}, c_{0}, P_{0}\right)$ s'écrit :

$$
\gamma\left(k_{0}, s_{0}, c_{0}, P_{0}\right):=\operatorname{Sup}_{k(.), s(.), c(.), P(.)} \operatorname{Inf}_{t \geq 0} \frac{d c(t) / d t}{c(t)}
$$

$\operatorname{Si} \gamma\left(k_{0}, s_{0}, c_{0}, P_{0}\right)$ est négatif, cela signifie qu'il n'est pas possible, à partir de la situation présente, de trouver une évolution viable où le niveau de consommation par tête est non décroissant pour au moins une génération ; mais on peut trouver l'évolution viable où cette baisse de consommation sera la plus faible.

Si $\gamma\left(k_{0}, s_{0}, c_{0}, P_{0}\right)$ est positif ou nul, cela signifie qu'il est possible, à partir de la situation présente, de trouver une évolution viable au cours de laquelle la consommation par habitant sera toujours croissante ou constante, son taux de croissance restant toujours supérieur ou égal à $\gamma\left(k_{0}, s_{0}, c_{0}, P_{0}\right)$.

À la différence de ce qui a été exposé dans la section précédente, où la borne inférieure $v_{\text {min }}$ était un paramètre fixé a priori, introduit comme contrainte sur l'évolution du taux de croissance de la consommation (Annexe A3), il s'agit ici de rechercher la plus grande valeur de $v_{\text {min }}$ telle qu'il existe une évolution viable où le taux de croissance de la consommation par tête sera supérieur ou égal à cette borne pour toutes les générations, en calculant le noyau de viabilité d'un nouveau métasystème de dimension supérieure (Annexe A4). Cette évolution viable est une évolution optimale, car, pour toutes les autres évolutions viables partant de cette situation initiale, une ou plusieurs générations auront nécessairement un taux de croissance de leur consommation par tête inférieur à la borne $v_{\min }=\gamma\left(k_{0}, s_{0}, c_{0}, P_{0}\right)$.

Il s'agit donc bien d'une méthode d'optimisation intertemporelle sélectionnant une évolution viable particulière de type "meilleur du pire des cas ». Ce mode de sélection d'une évolution optimale diffère d'un problème de contrôle optimal classique dans la mesure où le critère est un opérateur « sup inf » et non de type « intégral ». Par ailleurs, cette méthode d'optimisation ne nécessite pas que la fonction valeur soit différentiable et, surtout, elle prend en compte l'ensemble des contraintes sans conditions restrictives. Cette méthode diffère également des méthodes d'optimisation classiques dans le sens où le choix des contrôles peut être changé à chaque pas de temps pour prendre en compte les modifications des variables d'état et s'adapter aux contraintes de viabilité. Alors que les méthodes d'optimisation intertemporelles usuelles, par le biais de l'actualisation, cherchent à comparer et classer des évolutions où les décisions sont prises une fois pour toutes, cette approche s'apparente plus à un indicateur permettant à chaque génération de déterminer ses choix en fonction de ses contraintes et de ses capacités, pour s'engager sur un chemin de développement durable et équitable. Cette façon de sélectionner une évolution viable correspond à la définition du développement durable donnée par Asheim (2007) : «A generation's management of the resource base at some point in time is sustainable if it constitutes the first part of a feasible sustained development. A stream of wellbeing develops in a sustainable manner if each generation's management of the resource base is sustainable. »

\section{Conclusion}

Étudier le développement durable pose de nombreux problèmes théoriques et pratiques; la manière de les aborder est souvent limitée par les techniques disponibles, tandis que la manière de les évaluer est limitée par la disponibilité des informations adéquates. La découverte de nouvelles techniques permet parfois de renouveler la façon de penser ces problèmes. Ce fut le cas lorsque Weitzman (1976), en adaptant un formalisme plus général à ces modèles de croissance durable, a ouvert la voie à de nouvelles analyses et interprétations du développement durable, introduisant les concepts d'épargne véritable et de produit national vert. Les recherches actuelles sur la possibilité d'établir des indicateurs macroéconomiques de développement durable sont issues de ce changement.

La théorie de la viabilité commence à être utilisée avec un certain succès, en particulier dans le domaine de la gestion des ressources renouvelables. L'approche "viabilité », décrite comme une approche inverse permettant de trouver l'ensemble des évolutions respectant des contraintes à chaque moment plutôt que de rechercher des solutions réalisant un objectif établi a priori, est souvent confinée à la recherche de noyaux de viabilité. Elle est considérée comme une alternative à la recherche de solutions d'équilibre et comme opposée à la notion d'optimalité. Cet article sur les modèles de développement durable cherche à montrer que la viabilité ne se limite pas au concept de noyau de viabilité et au respect des contraintes. Les outils de la théorie de la viabilité n'interdisent pas forcément la recherche de solutions optimales, ils permettent aussi de résoudre des problèmes d'optimisation intertemporelle.

Pour nombre de chercheurs, la théorie de la viabilité est considérée comme une approche ouvrant la voie à un changement de paradigme, dans un domaine - l'environnement - où l'optique plus classique de recherche de trajectoires d'équilibre s'est révélée inadéquate. Surmonter ces difficultés ou ces insuffisances représente une 
véritable promesse qui doit être mesurée. La théorie de la viabilité est un outil puissant qui peut apporter des réponses à des questions précises issues d'un modèle bien formulé. Le spectre des questions peut être très large et ne se limite pas à celles concernant le «respect des contraintes », les problèmes de "pilotage » autonome sous contraintes sont un des fondements de cette théorie. L'arsenal mathématique de la théorie de la viabilité autorise l'étude et le calcul de nombreuses fonctions qui, correctement interprétées, peuvent être utiles pour étudier des aspects très divers des problèmes d'environnement et de développement durable. Mais concevoir des modèles pertinents pour des problèmes complexes est une tâche préalable qui peut être difficile.

L'utilisation des outils de la théorie de la viabilité se heurte à deux obstacles. D'une part, son maniement n'est pas aisé, il requiert un certain investissement dans ces techniques mathématiques non classiques et rarement enseignées, et la conception de modèles pertinents nécessite à la fois une bonne maîtrise du problème étudié et une connaissance minimale de ces techniques mathématiques. D'autre part, la dimension - et donc la complexité - des problèmes qu'il est possible d'étudier numériquement est actuellement encore limitée par la capacité des outils informatiques nécessaires pour effectuer des calculs sur des ensembles.

\section{Références}

Asheim, G.B., 2005. Intergenerational ethics under resource constraints, Swiss Journal of Economics and Statistics, 141, 313-330.

Asheim, G.B., 2007. Justifying, Characterizing and Indicating Sustainability, Dordrecht, Springer-Verlag.

Asheim, G.B., 2010. Intergenerational equity, Annual Review of Economics, 2, 197-222.

Aubin, J.-P., 1991. Viability Theory, Boston, Basel and Berlin, Birkhäuser.

Aubin, J.-P., 1996. Une métaphore mathématique du principe de précaution, Natures Sciences Sociétés, 4, 2, 146-154.

Aubin, J.-P., 1997. Dynamic Economic Theory: A Viability Approach, Berlin and New York, Springer-Verlag.

Aubin, J.-P., 2010. La Mort du devin, lémergence du démiurge: essai sur la contingence et la viabilité des systèmes, Paris, Beauchesne.

Aubin, J.-P., Bernardo, T., Saint-Pierre, P., 2004. A viability approach to global climate change issue, in Haurie, A., Viguier, L., The Coupling of Climate and Economic Dynamic: Essays on Integrated Assessment, Boston, Kluwers Academic Publishers, 1-31.

Aubin, J.-P., Saint-Pierre, P., 2005. Des «noyaux» dans les quotas de pêche, La Recherche, 385, 80-81.

Aubin, J.-P, Saint-Pierre, P., 2007. An introduction to viability theory and management of renewable resources, in Kropp, J., Scheffran, J. (Eds), Advanced Methods for Decision Making and Risk Management in Sustainability Science, Hauppauge (NY), Nova Science Publishers, 43-80.
Aubin, J.-P., Bayen, A., Saint-Pierre, P., 2011. Viability Theory: New Directions, Heidelberg and New York, SpringerVerlag.

Baumgärtner, S., Quaas, M.F., 2009. Ecological-economic viability as a criterion of strong sustainability under uncertainty, Ecological Economics, 68, 7, 2008-2020.

Béné, C., Doyen, L., Gabay, D., 2001. A viability analysis for a bio-economic model, Journal of Ecological Economics, 36, 3, 385-396.

Béné, C., Doyen, L., 2008. Contribution values of biodiversity to ecosystem performances: A viability perspective, Ecological Economics, 68, 1-2, 14-23.

Bernardo, T., 2008. Viabilité, analyse de sensibilité et mesures d'impact pour des systèmes dynamiques contraints : application à un modèle de changement climatique. Thèse de doctorat en sciences mathématiques, Université Paris-Dauphine, Paris.

Bonneuil, N., 2000. Viability in dynamic social networks, Journal of Mathematical Sociology, 24, 3, 175-182.

Bonneuil, N., 2003. Making ecosystem models viable, Bulletin of Mathematical Biology, 65, 1081-1094.

Bonneuil, N., Saint-Pierre, P., 2008. Beyond optimality: Managing children, assets, and consumption over the life cycle, Journal of Mathematical Economics, 44, 3-4, 227-241.

Dasgupta, P., Heal, G., 1974. The optimal depletion of exhaustible resources, The Review of Economic Studies, 41, 3-28.

De Lara, M., Doyen, L., Guilbaud, T., Rochet, M.-J., 2007. Is a management framework based on spawning-stock biomass indicators sustainable? A viability approach, ICES Journal of Marine Science, 64, 761-767.

De Lara, M., Doyen, L., 2008. Sustainable Management of Natural Resources: Mathematical Models and Methods, Springer.

Doyen, L., Béné, C., 2003. Sustainability of fisheries through marine reserve: A robust modelling analysis, Journal of Environmental Management, 69, 1, 1-13.

Doyen, L., De Lara, M., Ferraris, J., Pelletier, D., 2007. Sustainability of exploited marine ecosystem through protected areas: A viability model and a coreal reef case study, Ecological Modeling, 208, 2-4, 353-366.

Doyen, L., Dumas, P., Ambrosi, P., 2008. Optimal timing of $\mathrm{CO}_{2}$ mitigation policies for a cost-effectiveness model, Journal of Mathematics and Computer Modeling, 48, 882-897.

Eisenack, K., Scheffran, J., Kropp, J.-P., 2006. Viability analysis of management frameworks for fisheries, Environmental Modeling and Assessment, 11, 69-79.

Hartwick, J., 1977. Intergenerational equity and the investing of rents from exhaustible resources, American Economic Review, 97, 972-974.

Heal, G., 2005. Intertemporal welfare economics and the environment, in Mäler, K.-G., Vincent, J.R. (Eds), Handbook of Environmental Economics, vol. 3, 1105-1145.

Hotelling, H., 1931. The economics of exhaustible resources, Journal of Political Economy, 39, 137-175.

Koopmans, T.C., 1960. Stationary ordinal utility and impatience, Econometrica, 8, 2, 287-309.

Martin, S., 2004. The cost of restoration as a way of defining resilience: A viability approach applied to a model of lake eutrophication, Ecology and Society, 9, 2, 8.

Martin, S., 2005. La Résilience dans les modèles de systèmes écologiques et sociaux. Thèse de doctorat en mathématiques appliquées, ENS, Cachan. 
Martinet, V., Doyen, L., 2007. Sustainability of an economy with an exhaustible resource: A viable control approach, Resource and Energy Economics, 29, 17-39.

Martinet, V., Thébaud, O., Doyen, L., 2007. Defining viable recovery paths towards sustainable fisheries, Ecological Economics, 64, 2, 411-422.

Ramsey, F.P., 1928. A mathematical theory of savings, The Economic Journal, 38, 152, 543-559.

Rapaport, A., Terreaux, J.-P., Doyen, L., 2006. Viability analysis for the sustainable management of renewable resource, Journal of Mathematics and Computer Modelling, 43, 466-483.

Rawls, J, 1987. Théorie de la justice, Le Seuil. Éd. org. : Theory of Justice, Cambridge (MA), Belknap Press of Harvard University Press, 1971.

Saint-Pierre, P., 1994. Approximation of the viability kernel, Applied Mathematics E Optimisation, 29, 187-209.
Saint-Pierre, P., 1997. Le calcul du noyau de viabilité, outil $\mathrm{d}$ 'analyse des modèles dynamiques de systèmes contraints, in Blasco, F. (Ed.), Tendances nouvelles en modélisation pour l'environnement, Paris, Amsterdam et Oxford, Elsevier.

Solow, R.M., 1974. Intergenerational equity and exhaustible resources, The Review of Economic Studies, 41, 29-45.

Tichit, M., Hubert, B., Doyen, L., Genin, D., 2004. A viability model to assess the sustainability of mixed herds under climatic uncertainty, Animal Research, 53, 405-417.

Tichit, M., Doyen, L., Lemel, J.-Y., Renault, O., 2007. A coviability model of grazing and bird community management in farmland, Ecological Modelling, 206, 3-4, 277-293.

Weitzman, M.L., 1976. On the welfare significance of national product in a dynamic economy, The Quarterly Journal of Economics, 90, 1, 156-162.

\section{Annexes}

Un modèle de viabilité s'écrit sous la forme générale d'un système dynamique contrôlé et contraint :

$$
\text { (1) } \quad \forall t \geq 0 \quad\left\{\begin{array}{l}
x^{\prime}(t)=f(x(t), u(t)) \\
u(t) \in U(x(t)) \\
x(t) \in M
\end{array}\right.
$$

où $x^{\prime}(t)=\frac{d x(t)}{d t}=f(x(t), u(t))$ représente la dynamique du système dirigeant l'évolution des variables d'état $x(t)$ sous l'action des contrôles $u(t)$. Les contrôles $u$ appartiennent à un ensemble admissible qui dépend des variables d'état $U(x)$ et $M$ représente l'ensemble des contraintes sur l'état du système qui doivent être respectées pour tout $t \geq 0$.

Le noyau de viabilité de ce système est l'ensemble :

$$
\operatorname{Viab}_{f}(M):=\left\{x_{0} \in M \text { tel que } \exists u(.) \text { et } \exists x(.) \text { solution de (1) avec } x(0)=x_{0}\right\}
$$

Définitions des variables :

$P$ : population

$K$ : capital manufacturé

$S$ : ressource épuisable

$R$ : ressource totale extraite par période

$C$ : consommation

$n$ : taux de croissance de la population

$v$ : taux de croissance de la consommation par personne

$z$ : indicateur du niveau d'équité

On pose :

$k:$ capital manufacturé par personne $(k:=K / P)$

$c:$ consommation par personne $(c:=C / P)$

$r$ : quantité de ressource extraite par personne $(r:=R / P)$

$s:$ quantité de ressource disponible par personne $(s:=S / P)$

$s_{0}:$ quantité de ressource initiale disponible par personne

$s_{\mathrm{b}}$ : quantité de ressource par personne garantie à toutes les générations

$c_{\mathrm{b}}$ : consommation minimale garantie à toutes les générations 


\section{A1. Le modèle de Dasgupta et Heal (1974)}

Il correspond à un problème de contrôle optimal décrit par :

- une dynamique : $\left\{\begin{array}{l}d K(t) / d t=f(K(t), R(t))-C(t) \\ d S(t) / d t=-R(t)\end{array}\right.$

- un critère d'équité à maximiser : $\operatorname{Max} \int_{t}^{\infty} U(C(\tau)) e^{-\rho(t-\tau)} d \tau$

Le développement peut être durable si la fonction de production autorise la substitution entre les biens, par exemple une fonction de type Cobb-Douglas $f(K, R)=K^{\alpha} R^{\beta}$ avec $\alpha+\beta=1$ et $\alpha>\beta$.

A2.1. Durabilité/viabilité : respecter les contraintes en tout temps avec une population constante (Martinet et Doyen, 2007)

$$
\text { (2.1) } \forall t \geq 0 \quad\left\{\begin{array}{l}
x_{21}^{\prime}(t)=f_{21}\left(x_{21}(t), u_{21}(t)\right) \\
u_{21}(t) \in U_{21}\left(x_{21}(t)\right) \\
x_{21}(t) \in M_{21}
\end{array}\right.
$$

Variables d'état : $x_{21}:\{k, s\}$; variables de contrôle : $u_{21}:\{r, c\}$

Dynamique :

$$
\left\{\begin{array}{l}
\frac{d K(t)}{d t}=f(K(t), R(t))-C(t)=K(t)^{\alpha} R(t)^{\beta}-C(t) \\
\frac{d S(t)}{d t}=-R(t)
\end{array}\right.
$$

Relations caractérisant les contraintes sur les variables d'état: $M_{21}$

$\left\{\begin{array}{l}k \geq 0 \\ s \in\left[s_{b}, s_{0}\right]\end{array}\right.$

$$
\Leftrightarrow\left\{\begin{array}{l}
\frac{d k(t)}{d t}=k(t)^{\alpha} r(t)^{\beta}-c(t) \\
\frac{d s(t)}{d t}=-r(t)
\end{array}\right.
$$

Relations caractérisant les contraintes sur les variables de contrôle : $U_{21}$

$$
\left\{\begin{array}{l}
0 \leq r \leq r_{\max } \\
c_{b} \leq c \leq k^{\alpha} r^{\beta}
\end{array}\right.
$$

\section{A2.2. Durabilité/viabilité : prendre en compte une croissance exogène de la population}

$$
\text { (2.2) } \quad \forall t \geq 0\left\{\begin{array}{l}
x_{22}^{\prime}(t)=f_{22}\left(x_{22}(t), u_{22}(t)\right) \\
u_{22}(t) \in U_{22}\left(x_{22}(t)\right) \\
x_{22}(t) \in M_{22}
\end{array}\right.
$$

Variables d'état : $x_{22}:\{k, s\}$; variables de contrôle : $u_{22}:\{r, c\}$

Dynamique :

$$
\left\{\begin{array}{l}
\frac{d K(t)}{d t}=K(t)^{\alpha} R(t)^{\beta}-C(t) \\
\frac{d P(t)}{d t}=n P(t) \\
\frac{d S(t)}{d t}=-R(t)
\end{array}\right.
$$

Relations caractérisant les contraintes sur les variables d'état : $M_{22}$

$$
\left\{\begin{array}{l}
k \geq 0 \\
s \in\left[s_{b}, s_{0}\right]
\end{array}\right.
$$

$$
\Leftrightarrow \quad\left\{\begin{array}{l}
\frac{d k(t)}{d t}=k(t)^{\alpha} r(t)^{\beta}-n k(t)-c(t) \\
\frac{d P(t)}{d t}=n P(t) \\
\frac{d s(t)}{d t}=-r(t)-n s(t)
\end{array}\right.
$$

Relations caractérisant les contraintes sur les variables de contrôle : $U_{22}$

$$
\left\{\begin{array}{l}
0 \leq r \leq r_{\max } \\
c_{b} \leq c \leq k^{\alpha} r^{\beta} \\
n=0,05
\end{array}\right.
$$


A2.3. Durabilité/viabilité : introduire les fluctuations endogènes du taux de croissance de la population

$$
\text { (2.3) } \forall t \geq 0\left\{\begin{array}{l}
x_{23}^{\prime}(t)=f_{23}\left(x_{23}(t), u_{23}(t)\right) \\
u_{23}(t) \in U_{23}\left(x_{23}(t)\right) \\
x_{23}(t) \in M_{23}
\end{array}\right.
$$

Variables d'état : $x_{23}:\{k, s, P\}$; variables de contrôle : $u_{23}:\{r, c, n\}$

Dynamique :

$\left\{\begin{array}{ll}\frac{d K(t)}{d t}=K(t)^{\alpha} R(t)^{\beta}-C(t) \\ \frac{d P(t)}{d t}=n P(t) \\ \frac{d S(t)}{d t}=-R(t)\end{array} \quad \Leftrightarrow \quad\left\{\begin{array}{l}\frac{d k(t)}{d t}=k(t)^{\alpha} r(t)^{\beta}-n k(t)-c(t) \\ \frac{d P(t)}{d t}=n P(t) \\ \frac{d s(t)}{d t}=-r(t)-n s(t)\end{array}\right.\right.$

Relations caractérisant les contraintes sur les variables d'état : $M_{23}$

$$
\left\{\begin{array}{l}
k \geq 0 \\
s \in\left[s_{b}, s_{0}\right]
\end{array}\right.
$$

Relations caractérisant les contraintes sur les variables de contrôle : $U_{23}$

$$
\left\{\begin{array}{l}
0 \leq r \leq r_{\max } \\
c_{b} \leq c \leq k^{\alpha} r^{\beta} \\
n \in\left[n_{\min }, n_{\max }\right]
\end{array}\right.
$$

\section{A3. Durabilité/viabilité : imposer une limite au taux de croissance de la consommation par habitant}

$$
\text { (3) } \forall t \geq 0\left\{\begin{array}{l}
x^{\prime}{ }_{3}(t)=f_{3}\left(x_{3}(t), u_{3}(t)\right) \\
u_{3}(t) \in U_{3}\left(x_{3}(t)\right) \\
x_{3}(t) \in M_{3}
\end{array}\right.
$$

Variables d'état : $x_{3}:\{k, s, P, c\}$; variables de contrôle : $u_{3}:\{r, n, v\}$

Dynamique :

$$
\left\{\begin{array}{l}
\frac{d K(t)}{d t}=K(t)^{\alpha} R(t)^{\beta}-C(t) \\
\frac{d P(t)}{d t}=n(t) P(t) \\
\frac{d S(t)}{d t}=-R(t) \\
\frac{d C(t)}{d t}=(v(t)+n(t)) C(t)
\end{array}\right.
$$$$
\Leftrightarrow \quad\left\{\begin{array}{l}
\frac{d k(t)}{d t}=k(t)^{\alpha} r(t)^{\beta}-n(t) k(t)-c(t) \\
\frac{d P(t)}{d t}=n(t) P(t) \\
\frac{d s(t)}{d t}=-r(t)-n(t) s(t) \\
\frac{d c(t)}{d t}=v(t) c(t)
\end{array}\right.
$$

Relations caractérisant les contraintes sur les variables d'état: $M_{3}$

$$
\left\{\begin{array}{l}
k \geq 0 \\
s \in\left[s_{b}, s_{0}\right] \\
c \geq c_{b}
\end{array}\right.
$$

Relations caractérisant les contraintes sur les variables de contrôle : $U_{3}$

$$
\left\{\begin{array}{l}
0 \leq r(t) \leq r_{\text {max }} \\
n=\left[n_{\text {min }}, n_{\text {max }}\right] \\
v \geq v_{\text {min }} \\
c \leq k^{\alpha} r^{\beta}
\end{array}\right.
$$




\section{A4. Durabilité/viabilité : rechercher l'évolution viable la plus équitable}

$$
\text { (4) } \forall t \geq 0 \quad\left\{\begin{array}{l}
x_{4}^{\prime}(t)=f_{4}\left(x_{4}(t), u_{4}(t)\right) \\
u_{4}(t) \in U_{4}\left(x_{4}(t)\right) \\
x_{4}(t) \in M_{4}
\end{array}\right.
$$

Variables d'état : $x_{4}:\{k, s, P, c, z\}$; variables de contrôle : $u_{4}:\{r, n, v\}$

Dynamique :

$$
\left\{\begin{array}{l}
\frac{d K(t)}{d t}=K(t)^{\alpha} R(t)^{\beta}-C(t) \\
\frac{d P(t)}{d t}=n(t) P(t) \\
\frac{d S(t)}{d t}=-R(t) \\
\frac{d C(t)}{d t}=(v(t)+n(t)) C(t) \\
\frac{d z(t)}{d t}=0
\end{array}\right.
$$$$
\Leftrightarrow \quad\left\{\begin{array}{l}
\frac{d k(t)}{d t}=k(t)^{\alpha} r(t)^{\beta}-n(t) k(t)-c(t) \\
\frac{d P(t)}{d t}=n(t) P(t) \\
\frac{d s(t)}{d t}=-r(t)-n(t) s(t) \\
\frac{d c(t)}{d t}=v(t) c(t) \\
\frac{d z(t)}{d t}=0
\end{array}\right.
$$

Relations caractérisant les contraintes sur les variables d'état: $M_{4}$

$$
\left\{\begin{array}{l}
k \geq 0 \\
s \in\left[s_{b}, s_{0}\right] \\
c \geq c_{b}
\end{array}\right.
$$

Relations caractérisant les contraintes sur les variables de contrôle : $U_{4}$

$$
\left\{\begin{array}{l}
0 \leq r(t) \leq r_{\text {max }} \\
n=\left[n_{\text {min }}, n_{\text {max }}\right] \\
v \geq z \\
c \leq k^{\alpha} r^{\beta}
\end{array}\right.
$$

Reçu le 4 juin 2009. Accepté le 7 juin 2011. 